\title{
Non-Markovian qubit dynamics in a thermal field bath: Relaxation, decoherence and entanglement
}

\author{
S. Shresta C. Anastopoulos A A. Dragulescu团 and B. L. Hu团 \\ Department of Physics, University of Maryland, College Park, Maryland 20742
}

(Dated: October 10, 2018)

\begin{abstract}
We study the non-Markovian dynamics of a qubit made up of a two-level atom interacting with an electromagnetic field (EMF) initially at finite temperature. Unlike most earlier studies where the bath is assumed to be fixed, we study the coherent evolution of the combined qubit-EMF system, thus allowing for the back-action from the bath on the qubit and the qubit on the bath in a self-consistent manner. In this way we can see the development of quantum correlations and entanglement between the system and its environment, and how that affects the decoherence and relaxation of the system. We find non-exponential decay for both the diagonal and non-diagonal matrix elements of the qubit's reduced density matrix in the pointer basis. From the diagonal elements we see the qubit relaxes to thermal equilibrium with the bath. From the non-diagonal elements, we see the decoherence rate beginning at the usually predicted thermal rate, but changing to the zero temperature decoherence rate as the qubit and bath become entangled. These two rates are comparable, as was shown before in the zero temperature case [C. Anastopoulos and B. L. Hu, Phys. Rev. A 62 (2000) 033821]. On the entanglement of a qubit with the EMF under this type of resonant coupling we calculated, for the qubit reduced density matrix, the fidelity and the von Neumann entropy, which is a measure of the purity of the density matrix. The present more accurate non-Markovian calculations predict lower loss of fidelity and purity as compared with the Markovian results. Generally speaking, with the inclusion of quantum correlations between the qubit and its environment, the non-Markovian processes tend to slow down the drive of the system to equilibrium, prolonging the decoherence and better preserving the fidelity and purity of the system.
\end{abstract}

\section{INTRODUCTION}

Interest in quantum entanglement has grown in recent years motivated mainly by the attempt to understand and realize quantum information processing. An important aspect of quantum entanglement, which is unavoidable in quantum information processing, is the entanglement of a system with its environment. This issue is especially important to the feasibility of quantum computation, as the error threshold for fault tolerant error correction (without which quantum computation is impossible), is highly sensitive to the degree to which the environment becomes correlated with the qubits [1]. In many realistic quantum computing schemes the environment includes an electromagnetic field (EMF). Studies of entanglement between multi-partite systems exist 2, 3, 4, 5, [6], but few have attempted to predict the effects on a qubit from its entanglement with the EMF [7, 8, 9, 10]. We have addressed the relaxation and decoherence issues in various contexts, from a two-level atom in a (zero-temperature) EMF [11] to moving atoms in a cavity [12, 13]. Here we continue this study for these two issues and the issue of entanglement for a two-level atom in a finite temperature EMF.

In this paper we aim at addressing the issue of system-environment entanglement by carefully analyzing its effect on reduced system dynamics. Specifically, we study the reduced dynamics of a single qubit interacting with an initially thermal bath in the multimode Jaynes-Cummings model. The model is a well studied example of open system dynamics, however, prior analyses have focused on Markovian dynamics by assuming no disturbance of the bath modes by the qubit [14, 15, 16, 17, 18, 19. Although such a simplifying assumption does include a level of back-action, it neglects entanglement that forms between the qubit and bath modes during the evolution. Earlier predictions based on simplified Markovian approximations are thus unsuitable for studies of such entanglement, and should be scrutinized carefully before being applied to quantum computation (see e.g. 20] for a discussion of this issue bearing on error correction). More realistic physical conditions are better served by non-Markovian treatments, which have begun to appear. However, many of them use approximations with limited short-time validity [21, 22, 23, 24] or unclear physical meaning such as in non-Markovian stochastic Schroedinger equations [25, 26].

\footnotetext{
*Present Address: NIST, Atomic Physics Division, Gaithersburg, MD 20899-8423. Email: sanjiv.shresta@nist.gov

${ }^{\dagger}$ Present Address: Department of Physics, University of Patras, 26500 Patras, Greece. Email: anastop@physics.upatras.gr

‡Present Address: Constellation Energy Group, Baltimore, MD 21202. Email: adrian.dragulescu@constellation.com

§Email: hub@physics.umd.edu
} 
Our approach includes bath dynamics as well as qubit dynamics, and the quantum correlations between them. Analytic expressions are derived for the qubit reduced density matrix elements at low temperature, from which the fidelity (defined as the overlap between the initial and evolved qubit state) and von Neumann entropy are computed. Inspection of the matrix elements themselves show slowed (sub-exponential) decoherence for the off-diagonal elements and slowed relaxation to thermal asymptotes for the diagonal matrix elements, as compared to Markovian predictions. Analysis of the fidelity and von Neumann entropy similarly show slowed loss of fidelity and purity in the case of nonMarkovian dynamics over Markovian dynamics. The overall picture which develops is of increased preservation of coherence in non-Markovian dynamics.

Following successive degradation from an exact solution to a Markovian description of system-bath interactions, three distinct approximations are usually invoked. They are 1) the $2^{\text {nd }}$ order Born approximation, 2) the $1^{\text {st }}$ Markov approximation [19], and 3) the assumption of a bath which is unaffected by its interaction with the system. 1) The $2^{\text {nd }}$ order Born approximation is an approximation in the strength of the coupling constant, and applying it neglects terms of higher than $2^{\text {nd }}$ order in the coupling [27]. 2) The $1^{\text {st }}$ Markov approximation is an approximation in the back-action correlation time. It is a Markov approximation because it assumes that the back-action of the system onto itself through the bath at time $t$ will depend only on the state of the combined system-bath at time $t$, and not on the past history. It is called the $1^{\text {st }}$ Markov approximation because although it depends only on the state of the system at time $t$, it depends on the state of the bath as well as the system (through the bath averages), thus including the bath dynamics [19]. 3) The last of the above three approximations is the assumption of a bath state which is fixed for all time. That assumption expressly excludes any dynamical evolution of the bath.

In the usual derivation of the Markovian master equation from the Schrödinger equation for the system-bath density operator, all three approximations are made. In contrast, the Heisenberg-Langevin approaches make only the $1^{\text {st }}$ Markov approximation. However, for spin-boson models such approaches have focused on the reduced qubit dynamics in strictly vacuum EMF, although in the presence of a classical source (e.g. resonance florescence). The resulting equations for the qubit degrees of freedom are called the Bloch-Langevin equations [17]. The Schrödingermaster equation can be derived from the Heisenberg-Langevin equation after a perturbative expansion which imposes the first and third approximations from the above [28]. For a comparison of these approximations see Ref. [29]. Our path integral approach to the reduced system dynamics uses only the first of the above three approximations by allowing the combined system-bath to evolve coherently throughout the interaction period. Only at the end of all coherent evolution will the bath variables be traced out to yield the reduced system evolution.

The approach we take is straightforward, although the actual implementation includes some nonstandard techniques involving Grassmann path integrals. In Section II the Hamiltonian and other important aspects of the model, including the coherent state represenation, are reviewed. The transition amplitude is derived in Section IIIA, utilizing the coherent state representation for the bosonic degrees of freedom and Grassmann states for the qubit degrees of freedom, following [1]. Doing so will involve a recursive computation which exploits the semigroup property of the transition matrix (similar to a technique used in Ref. [30]). After evaluating the transition amplitudes in an intermediate form, we combine the forward and backward versions by tracing over the final bosonic coherent states to construct the reduced propagator in Section IIIB. An initial thermal state for the oscillator bath is then introduced and the reduced dynamics of the qubit are calculated in Section IIIC. Section IV gives discussion and further analysis of the results.

\section{MODEL}

\section{A. Hamiltonian}

The model used for atom-field interactions is the standard multi-mode Jaynes-Cummings model of a two-level system interacting with a harmonic oscillator bath. The total Hamiltonian under the dipole, rotating wave and two-level approximations is given by (e.g. Appendix A of [1]])

$$
H=\hbar \omega_{o} S_{z}+\hbar \sum_{k}\left[\omega_{k} b_{k}^{\dagger} b_{k}+\left(\lambda_{k} S_{+} b_{k}+\bar{\lambda}_{k} S_{-} b_{k}^{\dagger}\right)\right]
$$

where $\hat{b}_{k}^{\dagger}, \hat{b}_{k}$ are the creation and annihilation operators for the $k^{t h}$ bath mode with frequency $\omega_{k}$, and $\hbar \omega_{o}$ is the energy separation between the two levels. The operators $S_{z}, S_{+}$, and $S_{-}$are the qubit operators for z-projection, spin-up, and spin-down, respectively. The couplings, $\lambda_{k}$ and $\bar{\lambda}_{k}$, have absorbed a dependence on the spectral density of the bath [11]. 


\section{B. Coherent States}

Coherent states are defined as any set of states generated by the exponentiated operation of a creation operator and a suitable label on a chosen fiducial state [31, 32]:

$$
\begin{aligned}
\left|z_{k}\right\rangle & =\exp \left(z_{k} b_{k}^{\dagger}\right)\left|0_{k}\right\rangle \\
|\eta\rangle & =\exp \left(\eta S_{+}\right)|0\rangle
\end{aligned}
$$

In the case of the bosonic coherent states, defined in Eq. (2), the label $z_{k}$ is a complex number, and in the case of the Grassmann coherent states, defined in Eq. (3), the label $\eta$ is an anti-commuting number. The chosen fiducial states are the EMF vacuum and the lower state of the two-level system, respectively. A state of the combined atom-field system can be expanded in a direct product coherent state basis,

$$
\left|\left\{z_{k}\right\}, \eta\right\rangle=\left|\left\{z_{k}\right\}\right\rangle \otimes|\eta\rangle
$$

in which the bosonic coherent state basis, $\left|z_{k}\right\rangle$, is used to represent the EMF, and a Grassmann coherent state basis, $|\eta\rangle$, is used to represent the two-level internal degrees of freedom of the atom.

Grassmann and bosonic coherent states share well known properties of general coherent states, such as being non-orthogonal and eigenstates of the annihilator,

$$
\begin{array}{rlrl}
\left\langle\bar{z}_{k} \mid z_{k}^{\prime}\right\rangle & =\exp \left(\bar{z}_{k} z_{k}^{\prime}\right) & \left\langle\bar{\eta} \mid \eta^{\prime}\right\rangle=\exp \left(\bar{\eta} \eta^{\prime}\right) \\
b_{k}\left|z_{k}\right\rangle=z_{k}\left|z_{k}\right\rangle & S_{-}|\eta\rangle=\eta|\eta\rangle
\end{array}
$$

where the overbar denotes conjugation. Despite their non-orthogonality, both types of coherent states are (over)complete sets and have a resolution of unity,

$$
1=\int \mathrm{d} \mu\left(z_{k}\right)\left|z_{k}\right\rangle\left\langle\bar{z}_{k}\left|=\int \mathrm{d} \mu(\eta)\right| \eta\right\rangle\langle\bar{\eta}|
$$

with the measures

$$
\begin{aligned}
\mathrm{d} \mu\left(z_{k}\right) & =\exp \left(-\bar{z}_{k} z_{k}\right) \mathrm{d} \bar{z}_{k} \mathrm{~d} z_{k} \\
\mathrm{~d} \mu(\eta) & =\exp (-\bar{\eta} \eta) \mathrm{d} \bar{\eta} \mathrm{d} \eta
\end{aligned}
$$

That these measures are exponential functions makes the coherent states a particularly suitable representation for transition amplitudes written as path integrals. For convenience the short hand notation

$$
\prod_{k} \mathrm{~d} \mu\left(z_{k}\right)=\mathrm{d} \mu\left(\left\{z_{k}\right\}\right)
$$

is defined for the product of the measure of different mode coherent states.

In the bosonic and Grassmann coherent states, the Q-representation of the Hamiltonian, Eq. (1), is

$$
\left\langle\bar{\eta},\left\{\bar{z}_{k}\right\}|H| \eta^{\prime},\left\{z_{k}^{\prime}\right\}\right\rangle=\hbar \omega_{o} \bar{\eta} \eta^{\prime}+\hbar \sum_{k}\left[\omega_{k} \bar{z}_{k} z_{k}^{\prime}+\left(\lambda_{k} \bar{\eta} z_{k}^{\prime}+\bar{\lambda}_{k} \bar{z}_{k} \eta^{\prime}\right)\right]
$$

in which the replacement $S_{z} \rightarrow S_{+} S_{-}$, correct up to an additive constant, was made. The Q-representation Hamiltonian will participate prominently in the path integrals of the next section.

\section{APPROACH}

\section{A. Transition Amplitude}

Here we construct and evaluate the transition amplitude $K\left(t_{f}, t_{i}\right)$ of coherent states from an initial time, $t_{i}=0$, to coherent states at a final time, $t_{f}=t$,

$$
K(t, 0)=\left\langle\bar{\eta}_{f},\left\{\bar{z}_{f k}\right\} ; t|U(t, 0)| \eta_{i},\left\{z_{i k}\right\} ; 0\right\rangle .
$$


with $U(t, 0)$ being the time evolution operator,

$$
U(t, 0)=e^{-\frac{i}{\hbar} \int_{0}^{t} H \mathrm{ds}}
$$

Following the path integral methodology, we partition the interval $[0, t]$ into a large number $(N)$ of time steps, such that $t=N \epsilon$. The path integral is then calculated as a discrete functional. Doing so, the n-step transition amplitude can be written in a general form,

$$
K(n \epsilon, 0)=\exp \left\{\bar{\eta}_{n} \psi_{n}+\sum_{k} \bar{z}_{n k} f_{n k}+\sum_{k} \bar{\eta}_{n} g_{n k}+\sum_{k} \bar{z}_{n k} \phi_{n k}\right\} .
$$

By applying the semigroup property of the transition amplitude,

$$
K((n+1) \epsilon, 0)=\int \mathrm{d} \mu\left(\eta_{n}\right) \int \mathrm{d} \mu\left(\left\{z_{k}\right\}\right) K((n+1) \epsilon, n \epsilon) K(n \epsilon, 0)
$$

finite difference relations can be found for the coefficients in the action. Setting $h=1$, and absorbing factors of $2 \pi$, they are,

$$
\begin{array}{cl}
\psi_{n}=\left(1-i \omega_{o} \epsilon\right) \psi_{n-1}+\sum_{k}\left(i \lambda_{n, k} \epsilon\right) \phi_{n-1, k} & \psi_{0}=\eta_{i} \\
\phi_{n, k}=\left(i \bar{\lambda}_{n, k} \epsilon\right) \psi_{n-1}+\left(1-i \omega_{k} \epsilon\right) \phi_{n-1, k} & \phi_{0, k}=0 \\
& \\
g_{n, k}=\left(1-i \omega_{o} \epsilon\right) g_{n-1, k}+\left(i \lambda_{n, k} \epsilon\right) f_{n-1, k} & g_{0, k}=0 \\
f_{n, k}=\left(i \bar{\lambda}_{n, k} \epsilon\right) \sum_{\mathbf{l}} g_{n-1,1}+\left(1-i \omega_{k} \epsilon\right) f_{n-1, k} & f_{0, k}=z_{i, k} .
\end{array}
$$

The coupling constants in Eqs. (15 16) have time indices because they are separated by complete sets of states at different time steps when the Hamiltonian is partitioned, thus they are separate sets of Grassmann pairs. The transition amplitude at time $t(=N \epsilon)$ can be written:

$$
K(t, 0)=\exp \left\{\bar{\eta}_{f} \psi(t)+\sum_{k} \bar{z}_{f k} f_{k}(t)+\sum_{k} \bar{\eta}_{f} g_{k}(t)+\sum_{k} \bar{z}_{f k} \phi_{k}(t)\right\} .
$$

Since this equation is a function of Grassmann variables it is to be treated as a formal expression that has meaning only in its polynomial expansion. In that polynomial expansion many terms will be truncated due to the nilpotency of the Grassmann variables. Expanding out Eq. (17) and defining the functionals

$$
\begin{aligned}
F\left[\left\{m_{\xi}\right\}\right](t) & =\prod_{k}\left(f_{k}(t)\right)^{m_{k}} \\
G_{l}\left[\left\{m_{\xi}\right\}\right](t) & =g_{l}(t) \prod_{k}\left(f_{k}(t)\right)^{m_{k}} \\
\Psi^{f}\left[\left\{m_{\xi}\right\}\right](t) & =\psi(t) \prod_{k}\left(f_{k}(t)\right)^{m_{k}} \\
\Phi_{p}^{f}\left[\left\{m_{\xi}\right\}\right](t) & =\phi_{p}(t) \prod_{k}\left(f_{k}(t)\right)^{m_{k}} \\
\Phi_{l p}^{g}\left[\left\{m_{\xi}\right\}\right](t) & =g_{p}(t) \phi_{l}(t) \prod_{k}\left(f_{k}(t)\right)^{m_{k}} \\
\Psi_{p}^{g}\left[\left\{m_{\xi}\right\}\right](t) & =g_{p}(t) \psi(t) \prod_{k}\left(f_{k}(t)\right)^{m_{k}}
\end{aligned}
$$

gives the following expanded expression for the transition amplitude (with time dependence left implied for notational clarity)

$$
\begin{aligned}
K(t, 0)=\sum_{\left\{m_{\xi}\right\}}\left[\prod_{k} \frac{\left(\bar{z}_{f k}\right)^{m_{k}}}{m_{k} !}\right]\left(F\left[\left\{m_{\xi}\right\}\right]\right. & +\bar{\eta}_{f} \Psi^{f}\left[\left\{m_{\xi}\right\}\right]+\sum_{l} \bar{\eta}_{f} G_{l}\left[\left\{m_{\xi}\right\}\right] \\
& \left.+\sum_{p} \bar{z}_{f p} \Phi_{p}^{f}\left[\left\{m_{\xi}\right\}\right]+\sum_{p l} \bar{\eta}_{f} \bar{z}_{f p} \Phi_{l p}^{g}\left[\left\{m_{\xi}\right\}\right]\right)
\end{aligned}
$$


The variable $m_{\xi}$ is the number of photons in the $\xi^{\text {th }}$ mode of the final EMF state. The transition amplitude as written above is a functional sum over all distributions $\left\{m_{\xi}\right\}$. Differential equations for the functionals that appear in the transition amplitude can be found from the finite difference equations of Eqs. (15,16).

$$
\begin{aligned}
\dot{F}\left[\left\{m_{\xi}\right\}\right]= & -i \sum_{q} m_{q} \omega_{q} F\left[\left\{m_{\xi}\right\}\right]+i \sum_{l p} G_{p}\left[\left\{m_{\xi}-\delta_{\xi l}\right\}\right] \\
\dot{G}_{p}\left[\left\{m_{\xi}\right\}\right]= & -i\left(\omega_{o}+\sum_{q} m_{q} \omega_{q}\right) G_{p}\left[\left\{m_{\xi}\right\}\right]+i \lambda_{p} F\left[\left\{m_{\xi}+\delta_{\xi p}\right\}\right] \\
\dot{\Psi}^{f}\left[\left\{m_{\xi}\right\}\right]= & -i\left(\omega_{o}+\sum_{q} m_{q} \omega_{q}\right) \Psi^{f}\left[\left\{m_{\xi}\right\}\right]+i \sum_{p} \lambda_{p} \Phi_{p}^{f}\left[\left\{m_{\xi}\right\}\right] \\
& \quad+i \sum_{l p} m_{l} \lambda_{l} \Psi_{p}^{g}\left[\left\{m_{\xi}-\delta_{\xi l}\right\}\right] \\
\dot{\Psi}_{p}^{g}\left[\left\{m_{\xi}\right\}\right]= & -i\left(2 \omega_{o}+\sum_{q} m_{q} \omega_{q}\right) \Psi_{p}^{g}\left[\left\{m_{\xi}\right\}\right]-i \sum_{l} \lambda_{l} \Phi_{l p}^{g}\left[\left\{m_{\xi}\right\}\right] \\
\dot{\Phi}_{p}^{f}\left[\left\{m_{\xi}\right\}\right]= & -i\left(\omega_{p}+\sum_{q} m_{q} \omega_{q}\right) \Phi_{p}^{f}\left[\left\{m_{\xi}\right\}\right]+i \lambda_{p} \Psi^{f}\left[\left\{m_{\xi}\right\}\right] \\
& \quad+i \sum_{q l} m_{q} \lambda_{q} \Phi_{p l}^{g}\left[\left\{m_{\xi}-\delta_{\xi q}\right\}\right] \\
\dot{\Phi}_{l p}^{g}\left[\left\{m_{\xi}\right\}\right]= & -i\left(\omega_{o}+\omega_{l}+\sum_{q} m_{q} \omega_{q}\right) \Phi_{l p}^{g}\left[\left\{m_{\xi}\right\}\right]-i \lambda_{l} \Psi_{p}^{g}\left[\left\{m_{\xi}\right\}\right] \\
& \quad+i \lambda_{p} \Phi_{l}^{f}\left[\left\{m_{\xi}+\delta_{\xi p}\right\}\right]
\end{aligned}
$$

Although the transition amplitude of Eq. (24) and the differential equations with all Grassmann variables removed of Eqs. (2530) can be used from this point onward, it is simpler instead to work with Eq. (17) during the trace over final EMF states. In the next section we shall combine forward and backward versions of the transition amplitude to construct the reduced propagator.

\section{B. Reduced Propagator}

The evolution of the reduced system with an initial atomic state is given by,

$$
\rho(t)=\int d \mu\left(\eta_{i}\right) d \mu\left(\eta_{i}^{\prime}\right) \prod_{k}\left[d \mu\left(z_{i k}\right) d \mu\left(z_{i k}^{\prime}\right)\right] J_{R}(t, 0) R(0)
$$

in which $R(0)$ is the combined initial system-bath density operator and $J_{R}(t, 0)$ is the propagator for the reduced system,

$$
J_{R}(t, 0)=\int d \mu\left(\left\{z_{f k}\right\}\right) K(t, 0) \bar{K}^{\prime}(t, 0) .
$$

Carrying out the integration with Eq. (17) and its barred conjugate one finds,

$$
\begin{aligned}
J_{R}(t, 0)=\exp \left\{\bar{\eta}_{f} \psi(t)+\bar{\psi}^{\prime}(t) \eta_{f}^{\prime}\right. & +\sum_{k} \bar{\eta}_{f} g_{k}(t)+\sum_{k} \bar{g}_{k}^{\prime}(t) \eta_{f}^{\prime} \\
& \left.+\sum_{k}\left(\bar{f}_{k}^{\prime}(t)+\bar{\phi}_{k}^{\prime}(t)\right)\left(f_{k}(t)+\phi_{k}(t)\right)\right\}
\end{aligned}
$$




\section{Initial Thermal State}

For thermal vacuum the initial state in the coherent state representation and in units such that Boltzmann's constant is unity $\left(\mathrm{k}_{b}=1\right)$ is,

$$
R(0)=\left[\prod_{k} \exp \left\{e^{-\beta \omega_{k}} \bar{z}_{i k} z_{i k}^{\prime}\right\}\right] \times\left[\rho_{00}+\bar{\eta}_{i} \rho_{10}+\eta_{i}^{\prime} \rho_{01}+\bar{\eta}_{i} \eta_{i}^{\prime} \rho_{11}\right]
$$

Evaluating Eq. (31) with substitutions from Eq. (33) and Eq. (34) one may obtain the evolved reduced density operator. After expanding completely, the reduced density matrix elements become, for the upper state population,

$$
\begin{aligned}
& \rho_{11}(t)=\rho_{00} \sum_{\left\{m_{\xi}\right\}} \sum_{l} m_{l} G_{l}\left[\left\{m_{\xi}-\delta_{\xi l}\right\}\right] \bar{G}_{l}^{\prime}\left[\left\{m_{\xi}-\delta_{\xi l}\right\}\right] e^{-\beta \sum_{q} m_{q} \omega_{q}} \\
&+\rho_{11} \sum_{\left\{m_{\xi}\right\}}\left(\Psi^{f}\left[\left\{m_{\xi}\right\}\right]+\sum_{l} m_{l} \Phi_{l l}^{g}\left[\left\{m_{\xi}-\delta_{\xi l}\right\}\right]\right) \\
& \times\left(\bar{\Psi}^{\prime f}\left[\left\{m_{\xi}\right\}\right]+\sum_{l} m_{l} \bar{\Phi}_{l l}^{\prime g}\left[\left\{m_{\xi}-\delta_{\xi l}\right\}\right]\right) e^{-\beta \sum_{q} m_{q} \omega_{q}},
\end{aligned}
$$

for the lower state population,

$$
\begin{array}{r}
\rho_{00}(t)=\rho_{11} \sum_{\left\{m_{\xi}\right\}} \sum_{l}\left(m_{l}+1\right) \Phi_{l}^{f}\left[\left\{m_{\xi}\right\}\right] \bar{\Phi}_{l}^{\prime f}\left[\left\{m_{\xi}\right\}\right] e^{-\beta \sum_{q} m_{q} \omega_{q}} \\
+\rho_{00} \sum_{\left\{m_{\xi}\right\}} F\left[\left\{m_{\xi}\right\}\right] \bar{F}^{\prime}\left[\left\{m_{\xi}\right\}\right] e^{-\beta \sum_{q} m_{q} \omega_{q}}
\end{array}
$$

and for the off-diagonal,

$$
\rho_{10}(t)=\rho_{10} \sum_{\left\{m_{k}\right\}}\left(\Psi^{f}\left[\left\{m_{\xi}\right\}\right]+\sum_{l} m_{l} \Phi_{l l}^{g}\left[\left\{m_{\xi}-\delta_{\xi l}\right\}\right]\right) \bar{F}^{\prime}\left[\left\{m_{\xi}\right\}\right] e^{-\beta \sum_{q} m_{q} \omega_{q}}
$$

in terms of the definitions of Eqs. 18.23), with $\left\{\rho_{11}, \rho_{10}, \rho_{01}, \rho_{00}\right\}$ being the initial qubit density matrix elements.

\section{Low temperature}

The computation of the reduced density matrix elements involves the calculation of the functionals of Eqs. 18 . 231) and the evaluation of the functional summations in Eqs. (35.37). In order to calculate the functionals, a low temperature and a weak coupling approximation are applied to Eqs. (25 30). Details of the calculation are shown in Appendix A. The resulting expressions for the reduced density matrix elements, valid at low temperature $\left(\mathrm{e}^{-\beta \omega_{o}}<<1\right)$ and weak coupling $\left(\lambda^{2}<<1\right)$, are

$$
\begin{aligned}
& \rho_{11}(t)=[1-\Upsilon(t)] \rho_{00}+\left[1-\left(\frac{1-e^{-\Gamma_{o} t}}{1-e^{-\beta \omega_{o}-\Gamma_{o} t}}\right) \Upsilon(t)\right] \rho_{11} \\
& \rho_{00}(t)=\Upsilon(t) \rho_{00}+\left(\frac{1-e^{-\Gamma_{o} t}}{1-e^{-\beta \omega_{o}-\Gamma_{o} t}}\right) \Upsilon(t) \rho_{11} \\
& \rho_{10}(t)=\mathrm{e}^{-\Gamma_{o} t / 2-\mathrm{i} \omega_{o} t} \Upsilon(t) \rho_{10}
\end{aligned}
$$

with the definition

$$
\Upsilon(t)=\frac{1-e^{-\beta \omega_{o}}}{1-e^{-\beta \omega_{o}-\Gamma_{o} t}}
$$

and $\Gamma_{o}=\frac{2 \lambda^{2} \omega_{o}}{\pi}$ being the zero temperature spontaneous emission rate. These reduced density matrix elements are illustrated in Fig. (1). 

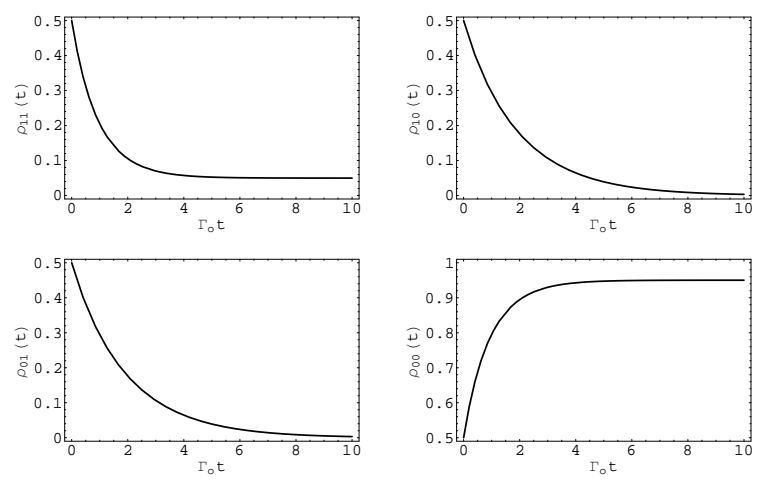

FIG. 1: These plots illustrate the non-Markovian reduced qubit matrix elements from Eqs. (38 41) for the case of an initial $\sigma_{x}$ eigenstate, $(|0\rangle+|1\rangle) / \sqrt{2}$ at low temperature $\left(\mathrm{e}^{-\beta \omega_{o}}=0.05\right)$, versus a dimensionless time in units of $\Gamma_{o}^{-1}$ where $\Gamma_{o}$ is the zero temperature spontaneous emission rate. The diagonal matrix elements thermalize to the low temperature values of Eqs. (42 43), and the off-diagonal matrix elements decohere non-exponentially.

In the long time limit the populations tend to the following thermal values valid at low temperature,

$$
\begin{aligned}
& \rho_{11}(t \rightarrow \infty)=\mathrm{e}^{-\beta \omega_{o}} \\
& \rho_{00}(t \rightarrow \infty)=1-\mathrm{e}^{-\beta \omega_{o}}
\end{aligned}
$$

and the off-diagonal coherence decays completely

$$
\rho_{10}(t \rightarrow \infty)=0
$$

\section{Zero temperature limit}

At zero temperature $\beta=\infty$ and Eqs. (3840) become,

$$
\begin{aligned}
& \rho_{11}(t)=\rho_{11} e^{-\Gamma_{o} t} \\
& \rho_{00}(t)=\rho_{00}+\rho_{11}\left(1-e^{-\Gamma_{o} t}\right) \\
& \rho_{10}(t)=\rho_{10} \mathrm{e}^{-\Gamma_{o} t / 2-\mathrm{i} \omega_{o} t}
\end{aligned}
$$

which is the expected result from Ref. [11].

\section{The Markov approximation limit}

For reference purposes we also include the results in the Markov approximation, which are valid in the regime of high temperature which are,

$$
\begin{aligned}
& \rho_{11}(t)=\rho_{11}(0) \mathrm{e}^{-\Gamma_{o} \operatorname{coth}\left(\beta \omega_{o} / 2\right) t}+\frac{\mathrm{e}^{-\beta \omega_{0}}}{1+\mathrm{e}^{-\beta \omega_{0}}}\left(1-\mathrm{e}^{-\Gamma_{o} \operatorname{coth}\left(\beta \omega_{o} / 2\right) t}\right) \\
& \rho_{00}(t)=1-\rho_{11}(t) \\
& \rho_{10}(t)=\rho_{10}(0) \mathrm{e}^{-i \omega_{o} t-\frac{\Gamma_{o} t}{2} \operatorname{coth}\left(\beta \omega_{o} / 2\right)} .
\end{aligned}
$$

Their asymptotic values are,

$$
\begin{aligned}
& \rho_{11}(t \rightarrow \infty)=\frac{\mathrm{e}^{-\beta \omega_{o}}}{1+\mathrm{e}^{-\beta \omega_{o}}} \approx \mathrm{e}^{-\beta \omega_{o}}+O\left(\left(\mathrm{e}^{-\beta \omega_{o}}\right)^{2}\right) \\
& \rho_{00}(t \rightarrow \infty)=\frac{1}{1+\mathrm{e}^{-\beta \omega_{o}}} \approx 1-\mathrm{e}^{-\beta \omega_{o}}+O\left(\left(\mathrm{e}^{-\beta \omega_{o}}\right)^{2}\right)
\end{aligned}
$$




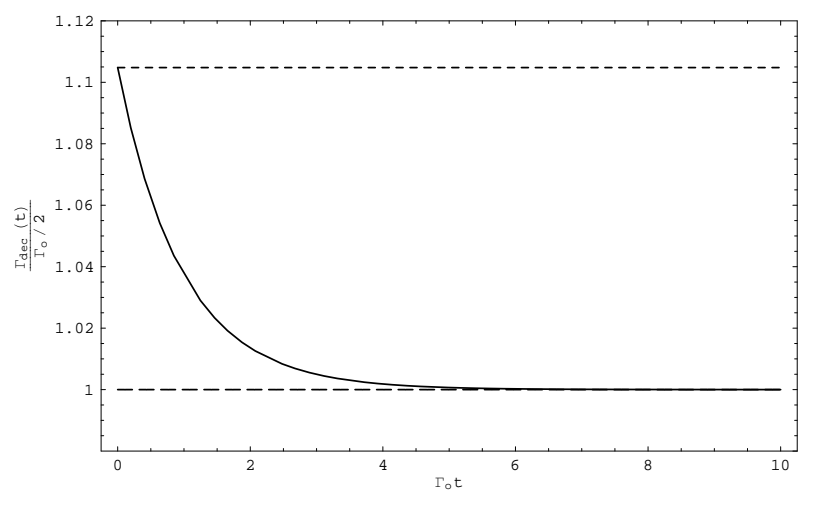

FIG. 2: This plot shows the quantity $\frac{\Gamma_{\text {dec }}(t)}{\Gamma_{o} / 2}$, (the ratio of the non-Markovian decoherence rate defined by $\Gamma_{d e c}(t):=-\frac{\dot{\rho}_{10}(t)}{\rho_{10}(t)}$, over the zero temperature decoherence rate, $\left.\Gamma_{o} / 2\right)$ versus the dimensionless time $\Gamma_{o} t$ in the low temperature regime, $e^{-\beta \omega_{o}}=0.05$. The dotted line is the value of the Markovian prediction at finite temperature, $\operatorname{coth}\left(\beta \omega_{o} / 2\right)$. Initially the non-Markovian prediction matches the Markovian result (at the dotted line). As the qubit and EMF become correlated the reduced dynamics deviates from the Markovian prediction and the decoherence rate asymptotes to the zero temperature value (along the dasheddotted line).

and the off-diagonal coherence decays completely

$$
\rho_{10}(t \rightarrow \infty)=0 .
$$

The thermal populations in the non-Markovian low temperature approximation match the Markovian thermal populations up to $O\left(\left(\mathrm{e}^{-\beta \omega_{o}}\right)^{2}\right)$.

\section{DISCUSSION}

\section{A. Decoherence}

The decoherence rate is found by computing the off-diagonal elements of the reduced density matrix (e.g. $\rho_{10}(t)$ ). The inclusion of bath as well as system dynamics causes the fall off of the off-diagonal matrix elements to become slightly sub-exponential. From previous work [1] we know that at zero temperature the decoherence rate is $\Gamma_{0} / 2=$ $\lambda^{2} \omega_{o} / \pi$. Markovian approaches (e.g. 14]) predict a decoherence rate of $\Gamma_{0} \operatorname{coth}\left(\beta \omega_{o} / 2\right) / 2$, valid at high temperatures. The present calculation shows that the decoherence rate, $\Gamma_{d e c}(t):=-\frac{\dot{\rho}_{10}(t)}{\rho_{10}(t)}$, actually changes as the total system evolves. As shown in Fig. (2) the decoherence rate at $t=0$, when the bath is by assumption in a thermal state uncorrelated with the qubit, agrees with the prediction of Markovian approaches. As the system and bath evolve together the decoherence rate falls back down to the zero temperature value. The interpretation of this is: initially the two cases have the same decoherence rate because by arrangement the combined system is a product state of qubit and thermal bath, which is the state assumed in Markovian approaches (there is no prior correlation). As the system and bath interact, the correlations that arise alter the reduced system dynamics and the combined state evolves away from that initial factorizable state. The overall effect is that the the qubit decoheres more slowly in non-Markovian dynamics than in Markovian dynamics.

\section{B. Relaxation}

The relaxation time scale is measured by the value of $\rho_{11}(t)$, assuming that $\rho_{11}(0)=1$. Similar to the case of decoherence, because the initial state of the combined system-bath is taken to be a product state of qubit and thermal bath, as it is in Markovian approaches, the dynamics for the populations initially agree in non-Markovian and Markovian dynamics (see the inset of Fig. (3)). Then as the system and bath interact, the non-Markovian result for the dynamics of the reduced system, which takes into consideration the dynamics of both the bath and the qubit, deviates from the Markovian prediction, as shown in Fig. (3). However, the long time behavior of our prediction matches the thermalization prediction of the Markovian prediction up to $O\left(\left(\mathrm{e}^{-\beta \omega_{o}}\right)^{2}\right)$. Most importantly, Fig. (3) shows that the upper state population relaxes more slowly in non-Markovian dynamics than in Markovian dynamics. 


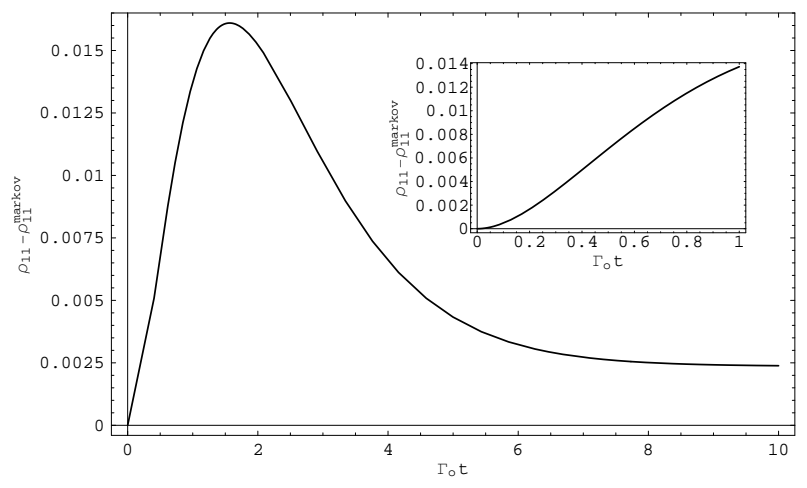

FIG. 3: This plot shows the difference in the upper state populations, $\rho_{11}(t)-\rho_{11}^{\text {markov }}(t)$, between the non-Markovian prediction, $\rho_{11}(t)$, and the Markovian prediction, $\rho_{11}^{\text {markov }}(t)$, given that the qubit is initially in the upper state (i.e. $\left.\rho_{11}(0)=1\right)$. The difference is plotted versus dimensionless time $\Gamma_{o} t$ and in the low temperature regime, $e^{-\beta \omega_{o}}=0.05$. Inspection of the plot shows that in non-Markovian dynamics the upper state decays more slowly than in Markovian dynamics. At long times the difference in the populations is zero up to $O\left(\left(\mathrm{e}^{-\beta \omega_{o}}\right)^{2}\right)$ (see Eq. (51)). The inset shows that the non-Markovian and Markovian predictions agree initially.

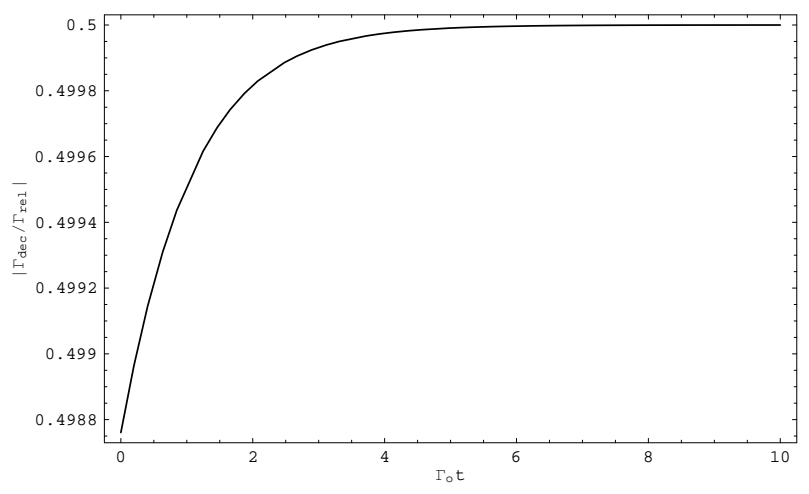

FIG. 4: This plot shows the ratio of the non-Markovian decoherence rate to the non-Markovian relaxation rate as a function of dimensionless time, $\Gamma_{o} t$, in the low temperature regime, $e^{-\beta \omega_{o}}=0.05$. The value is approximately constant at 0.5 , which is also the ratio between the Markovian decoherence and relaxation rates. The is because both cases share the same physical determining factor, i.e. that the resonant type of coupling is at work in this model.

We may define the relaxation rate (for the case that $\rho_{11}(0)=1$ ) as $\Gamma_{r e l}(t):=-\frac{\dot{\rho}_{11}(t)}{\rho_{11}(t)-\rho_{11}(\infty)}$. The plot of Fig. (4) shows the dependence of the ratio $\Gamma_{d e c}(t) / \Gamma_{r e l}(t)$ on time. It demonstrates that the relaxation and decoherence rate are of the same order of magnitude. In other words, the rate of quantum phase information escaping from the system to the environment is the same as the rate of energy flow. This property is characteristic of the resonant coupling between the two-level atom and the EMF, which leads to a different decoherence behavior from quantum Brownian motion (QBM) models [1]. One way to visualise the distinction is the realization that in QBM the couplings allow the interaction of the system with the far-infrared modes of the environment. The system then loses the phase information through soft photons which however carry very little energy. Hence in QBM systems, the relaxation time is much longer than the decoherence time. However, in resonant systems, such as being studied here and in [11], the system interacts primarily with the modes of the environment near the resonance frequency. Consequently, the phase information escapes through photons of energy equal to that of the atom and the decoherence rate is essentially the same with relaxation rate. We should remark that although the present results only hold for the low-temperature limit the near equality of decoherence and relaxation rate is valid even in the high temperature limit as can be seen already from the Markov approximation. 


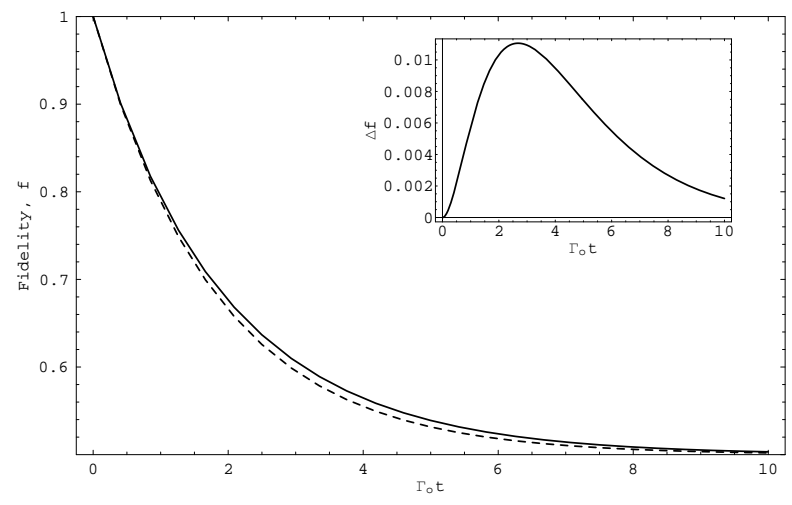

FIG. 5: This plot shows the quantity $\mathrm{f}=\operatorname{Tr}\left[\rho(t) U_{o}(t) \rho(0) U_{o}^{\dagger}(t)\right]$ as a function of the dimensionless time, $\Gamma_{o} t$, and at low temperature $\left(e^{-\beta \omega_{o}}=0.05\right)$ for an initial $\sigma_{x}$ eigenstate, $\left.(|0\rangle+|1\rangle) / \sqrt{2}\right)$, with $U_{o}(t)$ being the free evolution operator. Being a measure of the persistence of the initial qubit state after interaction with the environment, it can be considered as the fidelity of the qubit in its environment. The non-Markovian fidelity is plotted as a solid line and the Markovian fidelity is plotted as a dashed line. The inset is the non-Markovian fidelity minus the Markovian fidelity. Inspection of it shows that in the non-Markovian dynamics the EMF bath degrades the fidelity of the qubit more slowly than in the Markovian dynamics.

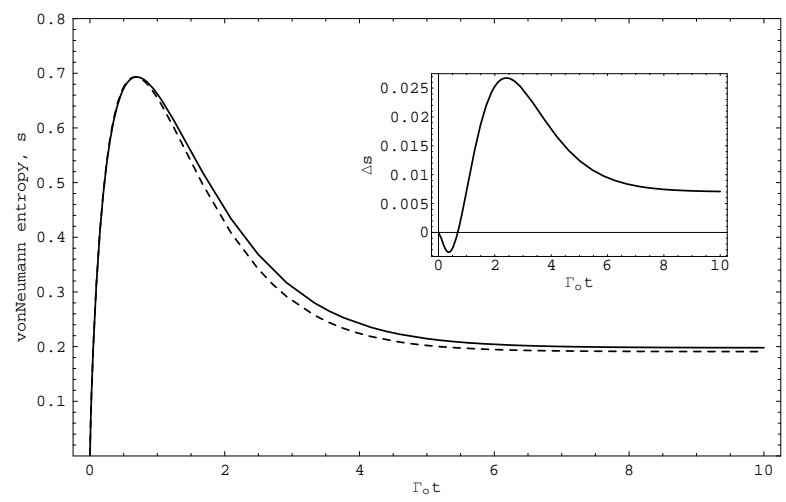

FIG. 6: This plot shows the von Neumann entropy, $s(t)=-\operatorname{Tr} \rho \log _{e}(\rho)$, of the reduced qubit density matrix versus the dimensionless time, $\Gamma_{o} t$, for low temperature $\left(e^{-\beta \omega_{o}}=0.05\right)$. The von Neumann entropy is a measure of the purity of a density operator. Both the non-Markovian and Markovian von Neumann entropies are plotted, as solid and dashed lines, respectively. Inspection of the plot shows that as the qubit interacts with the environment it becomes more mixed, but as the qubit equilibrates with the environment (after a time on the order of the relaxation time) it becomes less mixed due to the low temperature. In the inset the difference of the non-Markovian von Neumann entropy minus the Markovian von Neumann entropy is plotted. It shows that during the initial period of mixing non-Markovian dynamics predicts less mixing than does Markovian dynamics. Then, during the later equilibration period Markovian dynamics predicts a less mixed state. Finally, both dynamics reach a low temperature equilibrium state which is less mixed than at intermediate times. As in Fig. (3), the non-Markovian dynamics asymptotes to thermal equilibrium more slowly than the Markovian dynamics.

\section{Entanglement}

There exists no computable measure of entanglement between a qubit and an infinite continuous bath such as the electromagnetic field. However, since Markovian predictions explicitly exclude system-bath entanglement, comparison of those predictions with the present results can reveal the effects of system-bath entanglement. First is the decoherence rate discussed previously (see Fig. (2)). Its evolution from the thermal to the zero temperature value shows that the combined system-bath reaches and holds at some state in which the off-diagonal elements of the qubit are no longer affected by the thermal nature of the bath. No product state could give such behavior and cause thermalization of the populations. Second is the fidelity, $\mathrm{f}=\operatorname{Tr}\left[\rho(t) U_{o}(t) \rho(0) U_{o}^{\dagger}(t)\right]$, of the $(|0\rangle+|1\rangle) / \sqrt{2}$ state shown in Fig. (5), with $U_{o}(t)$ being the free evolution operator. The fidelity in this case is a measure of the persistence of the initial qubit state after interaction with the environment. Inspection of the difference between the non-Markovian and Markovian predictions for fidelity (inset of Fig. (5)) shows that non-Markovian dynamics predicts a slower loss of fidelity than Markovian dynamics, although with continued interaction both dynamics predict complete loss of fidelity. Third is the von Neumann entropy, $s(t)=-\operatorname{Tr} \rho \log _{e}(\rho)$, for the initial qubit state $|1\rangle$, shown in Fig. (6). 
The von Neumann entropy is a measure of the purity of a density matrix. Inspection of the difference in this case (inset of Fig. (6)) shows that the Markovian result initially predicts a greater loss of purity, but after a time on the order of the decay timescale, it predicts less loss of purity than the non-Markovian result. The reason for this seeming contradiction is that at low temperature, the system in its approach to thermal equilibrium, is driven to a less mixed state. Since correlations with the bath slow the drive to thermal equilibrium in non-Markovian dynamics, as shown in Fig. (3), this process is slower in the non-Markovian regime. Comparison of these three quantities (decoherence rate, fidelity and von Neumann entropy) shows a consistent picture in which non-Markovian dynamics is characterized by the preservation of coherence for longer time.

\section{Conclusion}

We have studied a two level atom coupled to an electromagnetic field (EMF) at finite temperature in the multimode Jaynes-Cummings model. We have computed the reduced evolution of the two level system and addressed the issues of decoherence, relaxation, and entanglement from its interaction with the EMF bath. Our approach makes use of a modified influence functional technique, which enables one to compute the reduced system dynamics while including the entangled evolution of the bath and qubit degrees of freedom. That is in contrast to Markovian approaches, which assume a fixed bath and hence neglect any dynamics in the bath. We adopt a Grassmann coherent state path integral representation for the atom degrees of freedom and bosonic coherent state path integral representation for the electromagnetic field, and assume a weak coupling ( $2^{\text {nd }}$ order Born) approximation under low temperature conditions.

We find non-exponential decay for both the diagonal and off-diagonal matrix elements of the qubit's reduced density matrix. From the diagonal elements we see the qubit relax to thermal equilibrium with the bath. From the off-diagonal elements, we see the decoherence rate beginning at the rate usually predicted for a thermal bath, but evolving to the zero temperature decoherence rate as the qubit and bath become entangled. Comparison of the relaxation and decoherence rates, shown in Fig. (4), reveals that as in the zero temperature case, both rates are comparable. At short times the ratio of the decoherence to the relaxation rate is initially smaller, but only by a small amount. At higher temperatures the initial difference between the two does increase, but that regime reaches the limits of validity of the present results. We can see why at low temperatures both rates are related to the atomic transition rate, because it is the only relevant physical scale present (unlike at finite temperature where the thermal scale is also at work). This, in turn, is a consequence of the particular resonant coupling between the two-level atom and the EMF, as explained earlier in [1].

On the entanglement of a qubit with the EMF (under resonant coupling) we calculated the qubit's fidelity and the von Neumann entropy. The Markovian result predicts higher loss of fidelity and purity as compared with the more accurate non-Markovian calculations. Qualitatively, with the inclusion of quantum correlations between the qubit and its environment, the non-Markovian processes tend to slow down the drive of the system to equilibrium, prolonging the decoherence and better preserving the fidelity and purity of the system.

Acknowledgements This work is supported in part by ARDA contract MDA90401/C0903, a NSF and a NIST grant to the University of Maryland.

[1] E. Knill, R. Laflamme and W. Zurek, Proc. R. Soc. Lond. A 454365 (1997); E. Knill, Keynote address given at First International Quantum Information and Quantum Control Conference, Fields Institute, University of Toronto, July 19-23, 2004 (http://www.fields.utoronto.ca/programs/scientific/04-05/quantumIC/abstracts/knill.pdf).

[2] M. Abdel-Aty, S. Furuichi and S. Nakamura, Quant. Inf. Comp. 2272 (2002)

[3] J. Eisert, C. Simon and M. B. Plenio, J. Phys. A: Math. Gen. 353911 (2002)

[4] M. Keyl, D. Schlingemann and R. F. Werner, Quant. Inf. Comp. 3281 (2003)

[5] G. K. Brennen, Quant. Inf. Comp. 3619 (2003); G. K. Brennen and S. S. Bullock, Phys. Rev. A quant-ph/0406064

[6] J. I. Latorre, E. Rico, and G. Vidal, Quant. Inf. Comp. 448 (2004)

[7] J. P. Barnes and D. J. Warren, Phys. Rev. A. 604363 (1999)

[8] J. Gea-Banacloche, Phys. Rev. A 65022308 (2002)

[9] S. J. van Enk and H. J. Kimble, Quant. Inf. Comp. 21 (2002)

[10] A. Silberfarb and I. H. S. Deutsch, Phys. Rev. A 69042308 (2004).

[11] C. Anastopoulos and B. L. Hu, Phys. Rev. A 62033821 (2000) quant-ph/9901078

[12] S. Shresta and B. L. Hu, Phys. Rev. A 68, 012110 (2003) quant-ph/0301180

[13] S. Shresta, B. L. Hu and N. G. Phillips, Phys. Rev. A 68, 062101 (2003) quant-ph/0302004

[14] H. J. Carmichael Statistical Methods in Quantum Optics 1: Master equations and Fokker-Planck Equations (Springer Berlin, 1999). 
[15] W. H. Louisell, Quantum Statistical Properties of Radiation (J Wiley and Sons, 1973).

[16] D. F. Walls and G. J. Milburn, Quantum Optics (Springer Verlag, Berlin, 1994).

[17] C. Cohen-Tannoudji, J. Dupont-Roc, and G. Grynberg, Atom-Photon Interactions: Basic Processes and Applications (J Wiley and Sons, 1992).

[18] O. Scully and M. Suhail Zubairy, Quantum Optics (Cambridge University Press, Cambridge, 1997).

[19] C. W. Gardiner and P. Zoller, Quantum Noise (Springer Verlag, Berlin, 2000).

[20] R. Alicki, M. Horodecki, P. Horodecki, and R. Horodecki, Phy. Rev. A 65062101 (2002).

[21] J. I. Kim, M. C. Nemes, A. F. R. de Toledo Piza and H. E. Borges, Phys. Rev. Lett. 77207 (1996).

[22] H. -P. Breuer, A. Ma and F. Petruccione, Time-local master equations: influence functional and cumulant expansion Quantum Computing and Quantum Bits in Mesoscopic Systems eds. Anthony Leggett, Berardo Ruggiero, Paolo Silvestrini (Kluwer Academic Plenum Publishers, 2003) quant-ph/0209153

[23] M. Esposito and P. Gaspard, Phys. Rev. A 68066112 (2003).

[24] D. Tolkunov and V. Privman, Phys. Rev. A 69062309 (2004); V. Privman, J. Stat. Phys. 110957 (2003); V. Privman, Mod. Phys. Lett. B 16459 (2002).

[25] J. Gambetta and H. Wiseman, Phys. Rev. A 66052105 (2002).

[26] T. Yu, quant-ph/0402086

[27] F. Haake, Z. Physik 223353 (1969).

[28] N. G. van Kampen, Stochastic Processes in Physics and Chemistry (Elsevier Science B. V., Amsterdam, 2003).

[29] S. Shresta, Ph. D. dissertation, University of Maryland 2003.

[30] I. M. Gelfand and A. M. Yaglom, J. Math Phys. 148 (1960).

[31] Y. Ohnuki and T. Kashiwa, Coherent states of Fermi operators and the path integral Coherent states: applications in physics and mathematical physics eds. J. Klauder and B. Skagerstam (Singapore: World Scientific 1978) 449-465.

[32] A. Perelomov, Generalized coherent states and their applications (Berlin: Springer 1986).

[33] J. Seke and W. N. Herfort, Phys Rev. A 38833 (1988)

[34] D. P. DiVincenzo and D. Loss, cond-mat/0405525

\section{APPENDIX A: CALCULATIONAL DETAILS OF QUBIT IN A THERMAL BATH}

\section{Approximated functional solutions}

Eqs. (25,30) are two sets of coupled differential equations. One set being the pair of equations

$$
\begin{aligned}
\dot{F}\left[\left\{m_{\xi}\right\}\right] & =-i \sum_{q} m_{q} \omega_{q} F\left[\left\{m_{\xi}\right\}\right]+i \sum_{l p} m_{l} \bar{\lambda}_{l} G_{p}\left[\left\{m_{\xi}-\delta_{\xi l}\right\}\right] \\
\dot{G}_{p}\left[\left\{m_{\xi}\right\}\right] & =-i\left(\omega_{o}+\sum_{q} m_{q} \omega_{q}\right) G_{p}\left[\left\{m_{\xi}\right\}\right]+i \lambda_{p} F\left[\left\{m_{\xi}+\delta_{\xi p}\right\}\right]
\end{aligned}
$$

and the remaining four equations comprising the other set. The solution method for this pair in the low temperature and weak coupling limits will be sketched out in this appendix. The solutions for the other set in the same limits will follow a similar sequence. First, given the initial conditions

$$
\begin{array}{r}
F\left[\left\{m_{\xi}\right\}\right](t=0)=1 \\
G_{p}\left[\left\{m_{\xi}\right\}\right](t=0)=0
\end{array}
$$

the Laplace transforms of the above equations are

$$
\begin{aligned}
z \tilde{F}\left[\left\{m_{\xi}\right\}\right](z)-1 & =-i \sum_{q} m_{q} \omega_{q} \tilde{F}\left[\left\{m_{\xi}\right\}\right](z)+i \sum_{l p} m_{l} \bar{\lambda}_{l} \tilde{G}_{p}\left[\left\{m_{\xi}-\delta_{\xi l}\right\}\right](z) \\
z \tilde{G}_{p}\left[\left\{m_{\xi}\right\}\right](z) & =-i\left(\omega_{o}+\sum_{q} m_{q} \omega_{q}\right) \tilde{G}_{p}\left[\left\{m_{\xi}\right\}\right](z)+i \lambda_{p} \tilde{F}\left[\left\{m_{\xi}+\delta_{\xi p}\right\}\right](z) .
\end{aligned}
$$

The second equation can be rearranged into

$$
\tilde{G}_{p}\left[\left\{m_{\xi}\right\}\right](z)=\frac{i \lambda_{p} \tilde{F}\left[\left\{m_{\xi}+\delta_{\xi p}\right\}\right](z)}{z+i\left(\omega_{o}+\sum_{q} m_{q} \omega_{q}\right)}
$$

which can be substituted back into Eq. A5 to give

$$
\left(z+i \sum_{q} m_{q} \omega_{q}\right) \tilde{F}\left[\left\{m_{\xi}\right\}\right](z)=1+i \sum_{l p} \frac{i m_{l} \bar{\lambda}_{l} \lambda_{p} \tilde{F}\left[\left\{m_{\xi}-\delta_{\xi l}+\delta_{\xi p}\right\}\right](z)}{z+i\left(\omega_{o}-\omega_{l}+\sum_{q} m_{q} \omega_{q}\right)} .
$$


In the expression above the low temperature approximation is applied by setting $p=l$ in the summation of the RHS. The justification is that the summation on the RHS will be peaked about $\omega_{l}=\omega_{o}$ such that the greatest contribution from $\tilde{F}\left[\left\{m_{\xi}-\delta_{\xi l}+\delta_{\xi p}\right\}\right](z)$ will be for $\omega_{l}=\omega_{o}$. However, at low temperatures those frequencies will not be populated. As a result the vacuum will be annihilated, unless $\delta_{\xi p}=\delta_{\xi l}$, which will cause the major contribution from the $p$ summation to be from $p=l$. The low temperature approximation is thus that the temperature is low enough that the modes with frequency $\omega_{o}$ are unoccupied, i.e. $\mathrm{e}^{-\beta \omega_{0}}<<1$. Applying this approximation, Eq. (A8) can be rewritten as

$$
\tilde{F}\left[\left\{m_{\xi}\right\}\right](z)=\left(z+i \sum_{q} m_{q} \omega_{q}+\sum_{l} \frac{m_{l} \lambda_{l}^{2}}{z+i\left(\omega_{o}-\omega_{l}+\sum_{q} m_{q} \omega_{q}\right)}\right)^{-1}
$$

The zero ${ }^{t h}$ order pole of $\tilde{F}\left[\left\{m_{\xi}\right\}\right](z)$ is at $z=-i \sum_{q} m_{q} \omega_{q}$. The reaction term at this point is found equal to $\frac{\Gamma_{o} m_{o}}{2}+i \Delta$, with $\Gamma_{o}=\frac{\lambda^{2} \omega_{o}}{\pi}$, which shows that the second order shift in the pole includes both a real and an imaginary part. After absorbing the imaginary part in a renormalization of the frequency, the second order pole is $z=-i \sum_{q} m_{q} \omega_{q}-\frac{\Gamma_{o} m_{o}}{2}$ with the definitions

$$
m_{o}=\sum_{\omega_{l}=\omega_{o}} m_{l}
$$

The desired functional can be calculated as in inverse Laplace transform of Eq. (A9) at the second order pole to give

$$
F\left[\left\{m_{\xi}\right\}\right](t)=\exp \left\{-\frac{\Gamma_{o} m_{o}}{2} t-i \sum_{q} m_{q} \omega_{q} t\right\}
$$

The inverse Laplace transform contains a contribution of a branch cut as well as a pole [33, 34]. We ignore the contribution of the branch cut, which is negligible at all but very late times such that $\Gamma_{o} t>20$ and very early times such that $\Gamma_{o} t<10^{-21}$ (see Eq.(3.20) of Ref. 33]). In all cases, we assume that time is much later than the inverse cut-off time. Further comparison between the branch cut and the non-Markovian correction over Markovian dynamics shows that the branch cut contribution is smaller by greater than three orders of magnitude for $\Gamma_{o} t>0.1$. The other functional in the pair can be calculated by integrating Eq. (26)

$$
G_{l}\left[\left\{m_{\xi}-\delta_{\xi l}\right\}\right](t)=i \frac{\lambda}{\sqrt{\omega_{l}}} \frac{1-e^{-\frac{\Gamma_{o} m_{o}}{2} t-i \sum_{q} m_{q} \omega_{q} t}}{\frac{\Gamma_{o} m_{o}}{2}+i\left(\omega_{l}-\omega_{o}\right)} e^{i\left(\omega_{l}-\omega_{o}-\sum_{q} m_{q} \omega_{q}\right) t} .
$$

Following similar calculations the rest of the functionals are found to be

$$
\begin{aligned}
& \Psi^{f}\left[\left\{m_{\xi}\right\}\right](t)=e^{-\frac{\Gamma_{o}\left(m_{o}+1\right)}{2} t-i\left(\omega_{o}+\sum_{q} m_{q} \omega_{q}\right) t} \\
& \Psi_{p}^{g}\left[\left\{m_{\xi}-\delta_{\xi p}\right\}\right](t)=\frac{\lambda e^{-\frac{\Gamma_{o}}{2} t-i\left(\omega_{o}+\sum_{q} m_{q} \omega_{q}\right) t}}{\sqrt{\omega_{p}}\left(\omega_{p}-\omega_{o}-i \frac{\Gamma_{o} m_{o}}{2}\right)}\left[e^{i\left(\omega_{p}-\omega_{o}\right) t}-e^{-\frac{\Gamma_{o} m_{o}}{2} t}\right] \\
& \Phi_{p}^{f}\left[\left\{m_{\xi}\right\}\right](t)=\frac{\lambda e^{-\frac{\Gamma_{o} m_{o}}{2} t-i\left(\omega_{o}+\sum_{q} m_{q} \omega_{q}\right) t}}{\sqrt{\omega_{p}}\left(\omega_{p}-\omega_{o}-i \frac{\Gamma_{o}}{2}\right)}\left[e^{-\frac{\Gamma_{o}}{2} t}-e^{i\left(\omega_{p}-\omega_{o}\right) t}\right] \\
& \Phi_{l p}^{g}\left[\left\{m_{\xi}-\delta_{\xi p}\right\}\right](t)=\frac{\lambda^{2} e^{-i\left(\omega_{o}+\omega_{l}-\omega_{p}+\sum_{q} m_{q} \omega_{q}\right) t}}{\sqrt{\omega_{l}} \sqrt{\omega_{p}}} \\
& \times \frac{\left(e^{-\frac{\Gamma_{o}}{2} t+i\left(\omega_{l}-\omega_{o}\right) t}-1\right)\left(1-e^{-\frac{\Gamma_{o} m_{o}}{2} t-i\left(\omega_{p}-\omega_{o}\right) t}\right)}{\left[\left(\omega_{l}-\omega_{o}\right)+i \frac{\Gamma_{o}}{2}\right]\left[\left(\omega_{p}-\omega_{o}\right)-i \frac{\Gamma_{o} m_{o}}{2}\right]}
\end{aligned}
$$

\section{Computation of density matrix elements}

The solutions of Eqs. (A11 A16) can be substituted into Eqs. (35.37) to evaluate the reduced density matrix elements in the limits of low temperature and weak coupling. The reduced density matrix elements in that form are summations over all distributions $\left\{m_{\xi}\right\}$. The $\rho_{10}(t)$ matrix element will be demonstrated below as a representative 
calculation. The evaluation of the other summations follow along similar lines. From Eq. (37), the off-diagonal density matrix element is

$$
\rho_{10}(t)=\rho_{10} \sum_{\left\{m_{\xi}\right\}}\left(\Psi^{f}\left[\left\{m_{\xi}\right\}\right]+\sum_{l} m_{l} \Phi_{l l}^{g}\left[\left\{m_{\xi}-\delta_{\xi l}\right\}\right]\right) \bar{F}^{\prime}\left[\left\{m_{\xi}\right\}\right] e^{-\beta \sum_{q} m_{q} \omega_{q}} .
$$

First, from Eqs. A11 A16 the functional in parentheses can be determined to be

$$
\Psi^{f}\left[\left\{m_{\xi}\right\}\right]+\sum_{l} m_{l} \Phi_{l l}^{g}\left[\left\{m_{\xi}-\delta_{\xi l}\right\}\right]=e^{-\frac{\Gamma_{o}\left(m_{o}+1\right)}{2} t-i\left(\omega_{o}+\sum_{q} m_{q} \omega_{q}\right) t},
$$

so that the off-diagonal matrix element becomes

$$
\rho_{10}(t)=\rho_{10} \sum_{\left\{m_{\xi}\right\}} \exp \left\{-\frac{\Gamma_{o}\left(2 m_{o}+1\right)}{2} t-i \omega_{o} t\right\} e^{-\beta \sum_{q} m_{q} \omega_{q}} .
$$

Denoting by primes those terms for which $\omega_{\xi}=\omega_{o}$ and double primes those for which $\omega_{\xi} \neq \omega_{o}$, the summand can be rewritten with the substitution $m_{o}=\sum_{\xi}^{\prime} m_{\xi}$,

$$
\rho_{10}(t)=\rho_{10} e^{-\frac{\Gamma_{o}}{2} t-i \omega_{o} t} \sum_{\left\{m_{\xi}\right\}} \prod_{\xi}^{\prime} e^{-\left(\Gamma_{o} t+\beta \omega_{o}\right) m_{\xi}} \prod_{\xi}^{\prime \prime} e^{-\beta \omega_{\xi} m_{\xi}} .
$$

The summation over distributions can be more clearly written as

$$
\sum_{\left\{m_{\xi}\right\}}=\left[\prod_{\xi} \sum_{m_{\xi}=0}^{\infty}\right]=\left[\prod_{\xi}^{\prime} \sum_{m_{\xi}=0}^{\infty}\right]\left[\prod_{\xi}^{\prime \prime} \sum_{m_{\xi}=0}^{\infty}\right],
$$

which allows us to bring Eq. A20 into the form

$$
\rho_{10}(t)=\rho_{10} e^{-\frac{\Gamma_{o}}{2} t-i \omega_{o} t}\left(\frac{1-e^{-\beta \omega_{o}}}{1-e^{-\left(\Gamma_{o} t+\beta \omega_{o}\right)}}\right) e^{-\sum_{q} \ln \left[1-e^{-\beta \omega_{q}}\right]}
$$

The factor at the end is removed by normalization of the reduced matrix element by its value if $\Gamma_{o}=0$. The final result for the off-diagonal matrix element is

$$
\rho_{10}(t)=\rho_{10} e^{-\frac{\Gamma_{o}}{2} t-i \omega_{o} t}\left(\frac{1-e^{-\beta \omega_{o}}}{1-e^{-\left(\Gamma_{o} t+\beta \omega_{o}\right)}}\right)
$$

with $\Gamma_{o}$ being the zero temperature spontaneous emission rate. The other reduced density matrix elements are given in the text. 